\title{
Metal free structural colours via disordered nanostructures with nm resolution and full CYMK colour spectrum
}

\author{
Marcella Bonifazi $^{a}$, Valerio Mazzone ${ }^{a}$ and Andrea Fratalocchi ${ }^{a}$ \\ ${ }^{a}$ PRIMALIGHT, King Abdullah University of Science and Technology (KAUST), Thuwal \\ 23955-6900, Saudi Arabia;
}

\begin{abstract}
Structural colours represents a research area of great interest, due to a wide field of application ranging from micro-security to biomimetic materials. At present metallic substrate are heavily employed and only a partial spectra of colours can be realised. We propose a novel, metal-free technology that exploits the complex scattering from a disordered three-dimensional dielectric material on a silicon substrate. We reproduce experimentally the full spectrum of CMYK colours, including variations in intensity. Our resolution lies in the nm range, limited only by the electron beam lithography fabrication process. We demonstrate that this technique is extremely robust, suitable for flexible and reusable substrates. Full of these notable proprieties these nano-structures fits perfectly with the requirements of a real-world technology.
\end{abstract}

Keywords: Structural colours, Silicon, metal-free, anti-counterfeiting, electron beam lithography, disorder, CMYK system, chromaticity diagram

\section{INTRODUCTION}

Colours play a vital role in our daily lives, both from a practical and psychological point of view and they have a very important role even in nature. ${ }^{1}$

Colours are used in the animal world for many different purposes; animals very often exploit colours typically to camouflage, signal, advertise or for temperature regulation. Animals colouration originates from different mechanisms such as pigments, variable colouration by means of chromatophores, bioluminescence and structural colouration. $^{2-9}$

If an object is illuminated with white light, a specific colour is perceived if it reflects to the observer a specific range window of wavelengths in the visible spectrum. In several millennia of evolution, nature has developed two main mechanisms in order to cut out specific wavelengths.

One option is absorption of light, common in materials such as pigments, dyes and metals.

The other possibility is to engineer a structure in such a way to remove specific range of wavelengths exploiting reflection, scattering and deflection of light. The latter has been subject of increasing attention by researchers in the last years, for its strong potential ranging from commercial to industrial and military applications. ${ }^{10-17}$ Structural colours are due to pure physical mechanisms connected with the complex interaction of light with micro and nanostructures. ${ }^{18}$

However all attempts to design artificial structural colours are based on expensive procedure and materials, such as metals and multi-layered structures.

For example, Phc (photonic crystals) are a promising platform to realise structural colors thanks to the peculiar scattering dynamics of light with a regularly arranged ensamble of particles. This structures are widely used in nature ${ }^{19}$ however is not possible to understand their real optical properties exclusively from bands calculation because it doesn't take into account defects and disorder. The state of the art technology is represented by the use of metallic periodic structures of $140 \mathrm{~nm}$, which achieve to create only a limited number of colors of the spectrum. ${ }^{20}$ Engineering the scattering from complex media already demonstrated to be successful in creating ultra-black material and new type of sources for optical applications. ${ }^{21}$

In this work we develop a novel technique based on random structure patterning by means of electron beam

Further author information: (Send correspondence to Andrea Fratalocchi)

Andrea Fratalocchi: E-mail: andrea.fratalocchi@kaust.edu.sa

Advanced Fabrication Technologies for Micro/Nano Optics and Photonics X, edited by Georg von Freymann,

Winston V. Schoenfeld, Raymond C. Rumpf, Proc. of SPIE Vol. 10115, 101150E

(C) 2017 SPIE $\cdot$ CCC code: 0277-786X/17/\$18 · doi: 10.1117/12.2251936

Proc. of SPIE Vol. 10115 101150E-1 


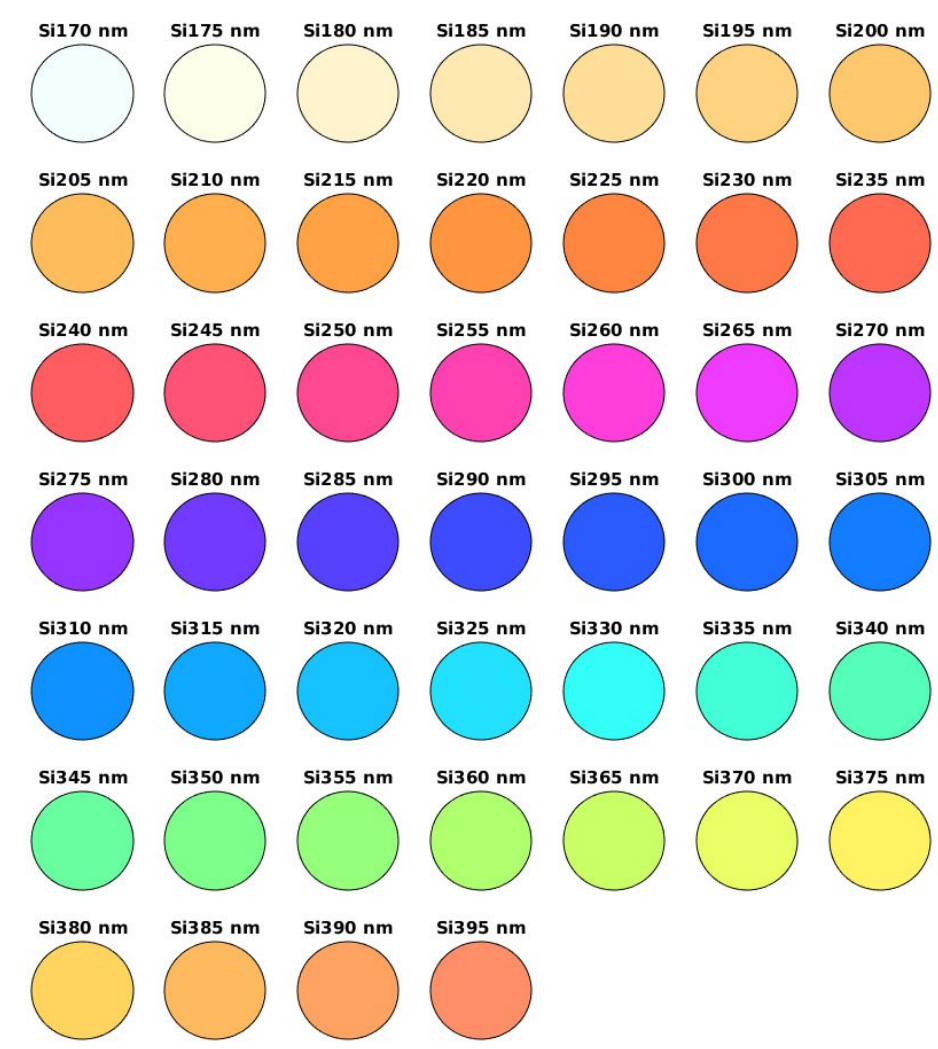

Figure 1. colours for different thickness of PMMA spin-coated on a Silicon substrate. The range of thickness goes from $170 \mathrm{~nm}$ to $395 \mathrm{~nm}$

lithography, able to generate all the fundamental colours with a simple, scalable, low cost and metal-free procedure. Our new approach realises the fundamental colors of the CMYK (Ciano, Magenta, Yellow and Black) system, and therefore is able to represent any color in the spectrum, including variations in intensity. The resolution of each pixel is limited by the EBL only, ranging in the nm scale. With this technique, we can print substrates as simple as silicon with the resolution of 158760 dpi. This method is interesting in the field of micro-security, due to the impossibility of counterfeiting a random, three-dimensional pattern of pixels created on a transparent dielectric material.

\section{FABRICATION PROCEDURE AND COLOURS}

The simplest physical phenomena to produce colours is by thin-film interference, but in this way is not possible to be able to reproduce the whole spectrum. To address this problem we decided to start our investigation with the calculation of the different chromaticities that could be obtained through thin-film interference. We use the method of the transfer matrix to simulate a layer of Poly Methyl Methacrlylate (PMMA) deposited on top of Silicon. The PMMA is a resist commonly used in electron beam lithography that, after being spin-coated and baked on an hot plate for around two minutes, forms an hard and transparent layer. Fig. 1 shows the calculated colours for different thickness of PMMA, spin-coated on a Silicon substrate, from $170 \mathrm{~nm}$ to $395 \mathrm{~nm}$, with $5 \mathrm{~nm}$ intervals. As shown in the figure 1, this method doesn't produce the whole spectrum and having a full palette of colours on the same sample by only thin film interference results impossible, from the fabrication point of view, due to the different thickness of PMMA resin needed to produce a single colour.

We have engineered our technique starting from a PMMA thickness of around $350 \mathrm{~nm}$.

The fabrication process is shown in Fig. 2. After the PMMA resist is spin-coated on a Silicon substrate, a pattern of random circles is transferred on the photoresist by means of electron-beam lithography (EBL). To 


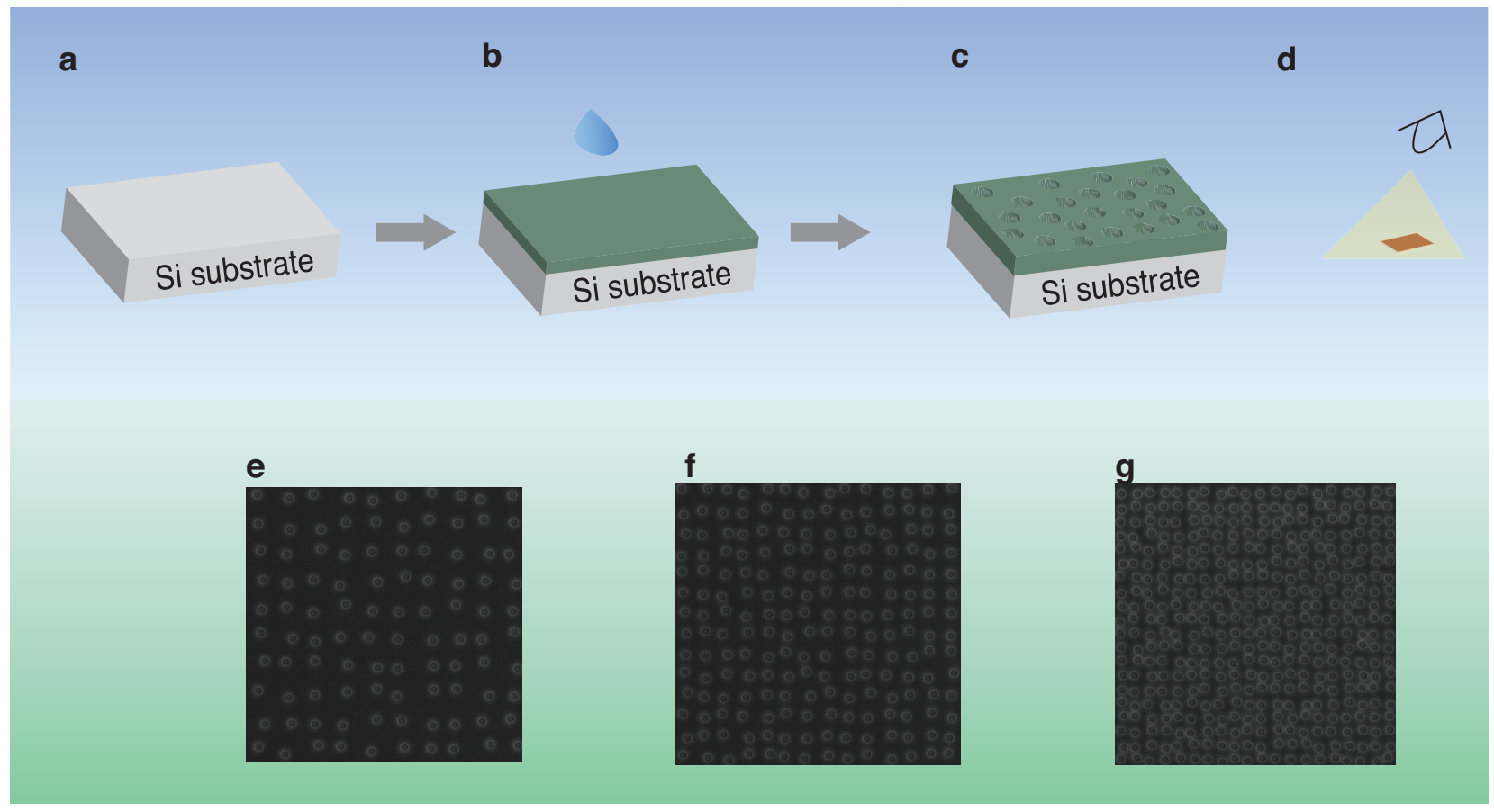

Figure 2. (a) Standard Silicon substrate used in the experiment, (b) spin-coating of the substrate with PMMA photoresist, (c) Electron-beam lithography is used to expose the random pattern of circles, (d) Visible coloration result after the pattern is developed in a MIBK solution, $(e, f, g)$ Typical SEM images of the resulting pattern for different filling fractions

obtain different colors starting from the same thickness of the resist we used different doses, that is, we exploited different exposure time to expose the substrate to the e-beam.

Once the sample is developed in a solution of MIBK 1:3 the exposed design transfer is completed and the colours become visible on the exposed zone of the photoresist.

In Fig. 2e-g a SEM image of the random pattern on the photoresist is presented, beside the precision of the pattern is not an issue, the filling fraction and the dimensions of the circles play the most important role to obtain different chromaticities. In order to change the colour perceived on the same substrate, we patterned different square zones of $20 \mu \mathrm{m}$ side, changing the writing parameters of the EBL, the radius of the circles and the filling fraction of the squared patterned areas. Through fine tuning all the variables we are able to obtain all the different chromaticities and all the shades, as shown in Fig. 3a and b. In these figures a sequence of colors is obtained by varying the filling fraction along the horizontal direction, with the structure already presented in Fig. $2 \mathrm{e}-\mathrm{g}$, and the doses increasing on the vertical direction. As it could be deduced, modifing the filling fraction only the shades change initially (left side of the figures), then we obtain also different chromaticities, such as going from blue to orange, passing through magenta and light purple (Fig.3a). Two different radius are presented in Fig 3a and b , $80 \mathrm{~nm}$ and $150 \mathrm{~nm}$ respectively.

In addition, as Fig. 3c confirms, mapping all the colours obtained on a chromaticity diagram, it is possible to notice that they are located all around the position where the white colour lies. Such a condition give us the possibility to generate the whole spectrum of colours, since it means that we are able to reproduce the chromaticities of the diagram only by patterning the PMMA surface with the EBL.

\section{CONCLUSIONS}

The absence of pigments in structural colours make them attractive for several reasons: they are environment friendly and they are robust and strong since they don't loose color under chemical or physical reactions.

Significant progress has been achieved recently in this field, however the necessity to look for massive-industrial applications, to cut down expensive fabrication procedure and the inability to obtain the whole spectrum deter- 
a

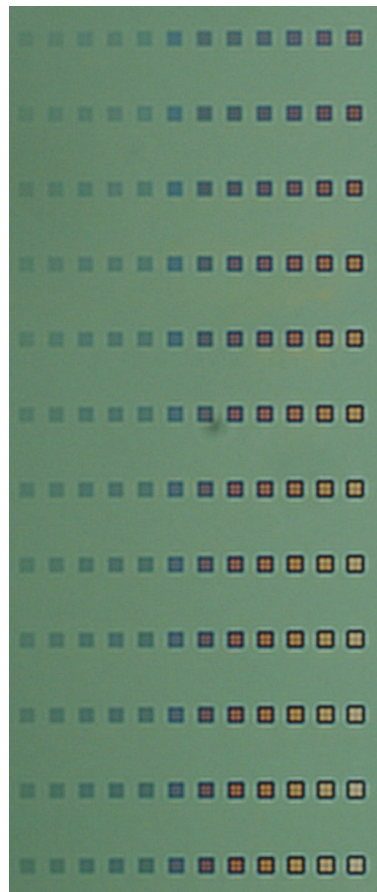

b

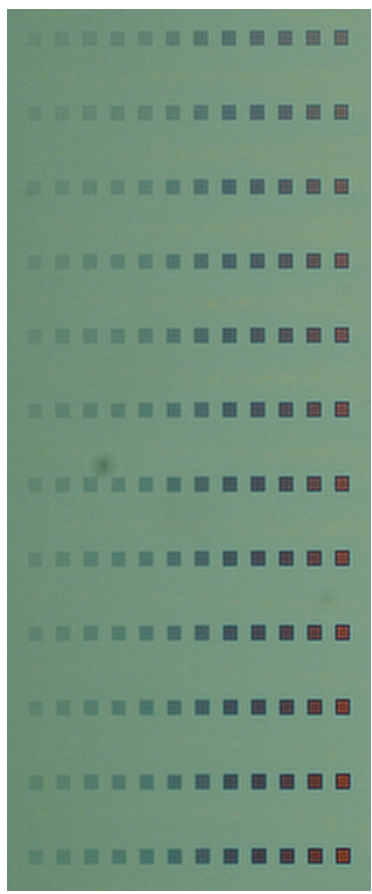

C

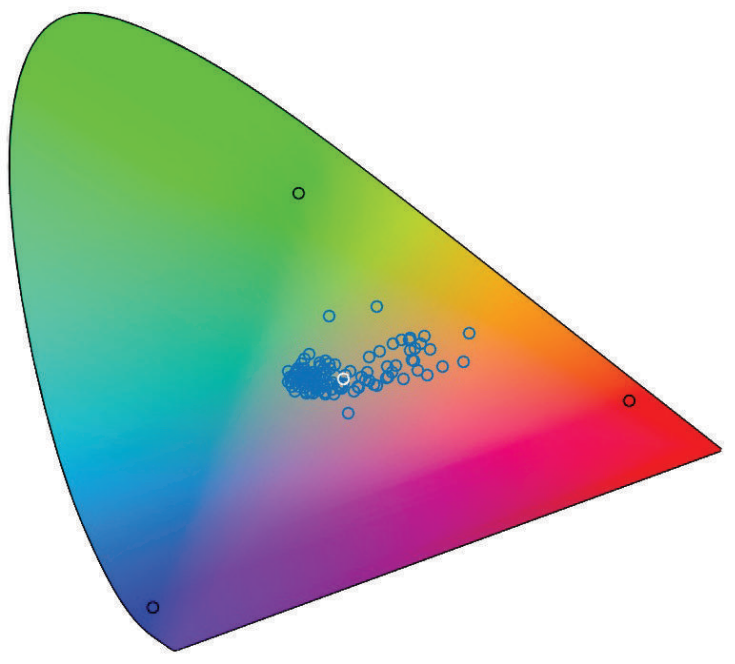

Figure 3. (a) Different filling fraction and doses for random patterns realised with circles of $80 \mathrm{~nm}$, (b) Different filling fraction and doses for random patterns realised with circles of $150 \mathrm{~nm}$, (c) Obtained colors mapped on a chromaticity diagram.

mine the need of further development. ${ }^{22}$

To this aim we have investigated the possibility to obtain colours with a scalable and totally metal-free technology, exploiting all the three physical phenomena involved in the structural colours generation, that are reflection, scattering and deflection of light. Through a simple fabrication process involving electron beam lithography we managed to obtain all the fundamental chromaticities in CYMK system, as well as all the other colors present in the chromaticity diagram. In addition to that, thanks to the disorder introduced in our pattern, resolution is not a an obstacle anymore, since we are able to reproduce very complicated shapes being limited only by the resolution of the machine involved in the fabrication process.

We have experimentally showed how, despite its simple geometry, tuning the random nano-structures fabricated in a standard photo-polymer, such as PMMA, unlocks the possibility to reproduce the full colour spectrum. In addition to that, this process could be easily scaled up thanks to other different lithography techniques, such as the nanoimprinting.

We therefore present the chance to obtain robust, scalable and cheap structural colours, implementing a procedure that opens up to a more massive spread of this kind of technology for numerous large scale industrial applications in different fields, from the anti-counterfeiting to the cladding for vehicles and buildings, through wearable biomimetic electronics. ${ }^{23-27}$

At the same time, another intriguing characteristic of this platform is the possibility to easily strip the PMMA and reuse the substrate for printing a new image.

\section{REFERENCES}

1. Parker, A. R., "515 million years of structural colour," Journal of Optics A: Pure and Applied Optics 2(6), R15 (2000).

2. Bradbury, J. W. and Vehrencamp, S. L., "Principles of animal communication," (1998). 
3. Potyrailo, R. A., Ghiradella, H., Vertiatchikh, A., Dovidenko, K., Cournoyer, J. R., and Olson, E., "Morpho butterfly wing scales demonstrate highly selective vapour response," Nature Photonics 1(2), 123-128 (2007).

4. Kinoshita, S., Yoshioka, S., and Miyazaki, J., "Physics of structural colors," Reports on Progress in Physics 71(7), 076401 (2008).

5. Teyssier, J., Saenko, S. V., Van Der Marel, D., and Milinkovitch, M. C., "Photonic crystals cause active colour change in chameleons," Nature communications 6 (2015).

6. Hooke, R., [Micrographia: or some physiological descriptions of minute bodies made by magnifying glasses, with observations and inquiries thereupon], Courier Corporation (2003).

7. Seago, A. E., Brady, P., Vigneron, J.-P., and Schultz, T. D., "Gold bugs and beyond: a review of iridescence and structural colour mechanisms in beetles (coleoptera)," Journal of the Royal Society Interface 6(Suppl 2), S165-S184 (2009).

8. Vukusic, P., Hallam, B., and Noyes, J., "Brilliant whiteness in ultrathin beetle scales," Science 315(5810), 348-348 (2007).

9. Dyck, J., "Structure and colour-production of the blue barbs of agapornis roseicollis and cotinga maynana," Zeitschrift für Zellforschung und Mikroskopische Anatomie 115(1), 17-29 (1971).

10. Kats, M. A., Blanchard, R., Genevet, P., and Capasso, F., "Nanometre optical coatings based on strong interference effects in highly absorbing media," Nature materials 12(1), 20-24 (2013).

11. Kats, M. A., Sharma, D., Lin, J., Genevet, P., Blanchard, R., Yang, Z., Qazilbash, M. M., Basov, D., Ramanathan, S., and Capasso, F., "Ultra-thin perfect absorber employing a tunable phase change material," Applied Physics Letters 101(22), 221101 (2012).

12. Schlich, F. F. and Spolenak, R., "Strong interference in ultrathin semiconducting layers on a wide variety of substrate materials," Applied Physics Letters 103(21), 213112 (2013).

13. Zhang, Y., Dong, B., Chen, A., Liu, X., Shi, L., and Zi, J., "Using cuttlefish ink as an additive to produce non-iridescent structural colors of high color visibility," Advanced Materials 27(32), 4719-4724 (2015).

14. Shen, Y., Rinnerbauer, V., Wang, I., Stelmakh, V., Joannopoulos, J. D., and Solja?i?, M., "Structural colors from fano resonances," ACS Photonics 2(1), 27-32 (2015).

15. Gu, Y., Zhang, L., Yang, J. K., Yeo, S. P., and Qiu, C.-W., "Color generation via subwavelength plasmonic nanostructures," Nanoscale 7(15), 6409-6419 (2015).

16. Forster, J. D., Noh, H., Liew, S. F., Saranathan, V., Schreck, C. F., Yang, L., Park, J.-G., Prum, R. O., Mochrie, S. G., O'Hern, C. S., et al., "Biomimetic isotropic nanostructures for structural coloration," Advanced Materials 22(26-27), 2939-2944 (2010).

17. Antosiewicz, T. J., Apell, S. P., Zäch, M., Zorić, I., and Langhammer, C., "Oscillatory optical response of an amorphous two-dimensional array of gold nanoparticles," Physical review letters 109(24), 247401 (2012).

18. McPhedran, R. C., Nicorovici, N., McKenzie, D., Rouse, G. W., Botten, L., Welch, V., Parker, A., Wohlgennant, M., and Vardeny, V., "Structural colours through photonic crystals," Physica B: Condensed Matter 338(1), 182-185 (2003).

19. Zi, J., Yu, X., Li, Y., Hu, X., Xu, C., Wang, X., Liu, X., and Fu, R., "Coloration strategies in peacock feathers," Proceedings of the National Academy of Sciences 100(22), 12576-12578 (2003).

20. Kumar, K., Duan, H., Hegde, R. S., Koh, S. C., Wei, J. N., and Yang, J. K., "Printing colour at the optical diffraction limit," Nature Nanotechnology 7(9), 557-561 (2012).

21. Huang, J., Liu, C., Zhu, Y., Masala, S., Alarousu, E., Han, Y., and Fratalocchi, A., "Harnessing structural darkness in the visible and infrared wavelengths for a new source of light," Nature nanotechnology (2015).

22. Galinski, H., Favraud, G., Dong, H., Gongora, J. S. T., Favaro, G., Döbeli, M., Spolenak, R., Fratalocchi, A., and Capasso, F., "Scalable, ultra-resistant structural colors based on network metamaterials," arXiv preprint arXiv:1605.03700 (2016).

23. Berthier, S., Boulenguez, J., and Bálint, Z., "Multiscaled polarization effects in suneve coronata (lepidoptera) and other insects: application to anti-counterfeiting of banknotes," Applied Physics A 86(1), 123-130 (2007).

24. Hu, H., Chen, Q.-W., Tang, J., Hu, X.-Y., and Zhou, X.-H., "Photonic anti-counterfeiting using structural colors derived from magnetic-responsive photonic crystals with double photonic bandgap heterostructures," Journal of Materials Chemistry 22(22), 11048-11053 (2012). 
25. Parker, A. R. and Townley, H. E., "Biomimetics of photonic nanostructures," Nature nanotechnology 2(6), 347-353 (2007).

26. Wu, Y.-K. R., Hollowell, A. E., Zhang, C., and Guo, L. J., "Angle-insensitive structural colours based on metallic nanocavities and coloured pixels beyond the diffraction limit," Scientific reports 3, 1194 (2013).

27. Yoon, B., Lee, J., Park, I. S., Jeon, S., Lee, J., and Kim, J.-M., "Recent functional material based approaches to prevent and detect counterfeiting," Journal of Materials Chemistry C 1(13), 2388-2403 (2013). 